\title{
Adhesion G-Protein Coupled Receptor G1
}

National Cancer Institute

\section{Source}

National Cancer Institute. Adhesion G-Protein Coupled Receptor G1. NCI Thesaurus.

Code C99659.

Adhesion G-protein coupled receptor G1 (693 aa, 78 kDa) is encoded by the human

ADGRG1 gene. This protein is involved in G protein-coupled receptor signaling, which may be dependent on the removal of its extracellular domain. 